\title{
Transient Vibration in an Airplane Wing Obtained by Several Methods ${ }^{1}$
}

\author{
By Walter Ramberg
}

\begin{abstract}
Analysis of the flexural transients in a model airplane wing following a "soft" unsymmetrical two-point landing impact indicates that Williams' method, separating "static" and vibrational response, is superior to the normal modes method used by Biot and Bisplinghoff, in leading to values in good agreement with experiment without using more than four modes. A method of coupled modes suggested by Levy leads to values of the response about as accurate as those from Williams' method with as few as three vibrational modes.

None of the three methods showed rapid convergence for the response following a "hard" impact of the same shape as the soft impact, but with only one-fifth of the duration. Attempts to develop a traveling wave method for the solution of this problem have been unsuccessful so far.
\end{abstract}

\section{Statement of Problem}

The problem of determining the transients in an airplane following the landing impact has become important during recent years with the advent of large transport airplanes.

It became apparent, as a result of several failures in service, that the stresses developed during the landing of large transport airplanes could be far in excess of those computed from the characteristics of the landing gear on the assumption that the airplane was decelerated a a rigid body during the landing impact. It was obvious that an adequate stress analysis of the airplane would have to take into account the transients excited by the landing impact. Several methods were soon proposed for computing these transients, notably the statistical method of Biot and Bisplinghoff $[1],{ }^{2}$ in which the response of the airplane for impacts of standard shapes is resolved in terms of the normal modes of vibration of the airplane, and the method of David Williams [2], in which

${ }_{1}^{1}$ Paper presented before Seventh International Congress of Applied Mechanics, London, September 1948.

The work described in this paper was conducted for the Bureau of Aeronauties, Navy Department.

2 Figures in brackets indicate the literature references at the end of this paper. the static response and the vibrational response are computed separately. A method of coupled modes has been suggested recently by Levy, in which the modes are computed directly from the rigid body motion. Finally, there is a possibility of adapting the method of traveling waves that was used by St. Venant over 60 years ago to soive the problem of longitudinal impact of an elastic bar.

It was decided to try out these methods in the computation of flexural transients in an airplane wing following a relatively soft and a relatively hard landing impact. It was hoped that the computations would indicate the effectiveness of these methods for the rapid and accurate computation of transients in an airplane wing.

\section{Methods Tried}

\section{Method of Normal Modes (Biot and Bisplinghoff)}

The method of normal modes for computing the response of an elastic structure to impact is described in detail in the classical paper of Biot and Bisplinghoff [1], referred to above. The method makes use of the independence of the motion in one normal mode from that in any other normal mode. 
The total displacement $y(x, t)$ of any point with coordinate $x$ is then given as the sum of displacements $y_{i}(x, t)$ in each one of the modes $i$ that may be excited by the impact

$$
y(x, t)=\sum_{i=1}^{\infty} y_{i}(x, t)
$$

The displacement $y_{i}(x, t)$ in the $i$ th mode can be derived from the displacement $q_{i}(t)$ of the linear

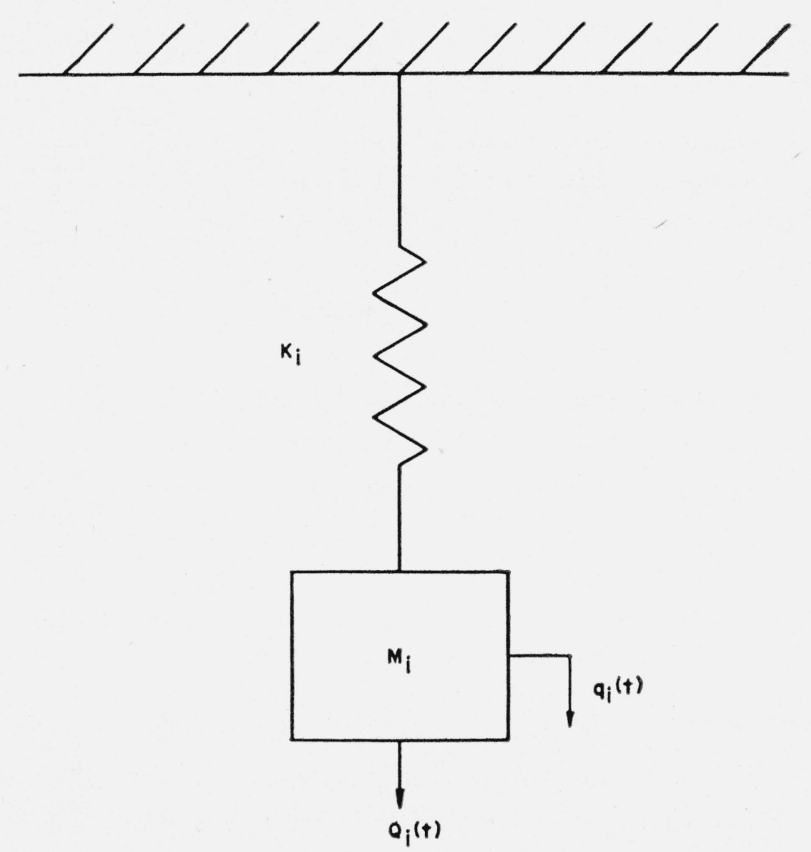

Figure 1. Equivalent linear oscillator.

oscillator of figure 1 in accordance with the equation

where

$$
y_{i}(x, t)=\varphi_{i}(x) q_{i}(t),
$$

$\varphi_{i}(x)=$ normalized deflection in $i$ th mode, corresponding to unit deflection of the reference station, e. g., the wing tip; $q_{i}(t)=$ deflection at reference station.

The deflection $q_{i}(t)$ of the linear oscillator of figure 1 under the action of an external force $Q_{i}(t)$ is given by the well-known integral of Duhamel:

$$
q_{i}(t)=\frac{1}{M_{i} \omega_{i}} \int_{0}^{i} Q_{i}(\tau) \sin \omega_{i}(t-\tau) d \tau .
$$

It is shown in reference [1] that the deflection $q_{i}(t)$ has the correct value for the $i$ th mode of the elastic structure with its distributed mass $m(x)$ under the action of an external impact force $F\left(x_{f}, t\right)$ if

$$
\left.\begin{array}{rl}
M_{i}= & \int \varphi_{i}^{2}(x) d m=\text { generalized mass in } i \text { th } \\
& \text { mode; } \\
\omega_{i}= & \sqrt{K_{i} / M_{i}}=\text { frequency of } i \text { th mode, } \\
& \text { radians } / \mathrm{sec} ; \\
Q_{i}(t)= & \varphi_{i}\left(x_{f}\right) F\left(x_{f}, t\right)=\text { generalized force in } \\
& i \text { th mode. }
\end{array}\right\}
$$

The stress at any station $x$ is obtained by superposing the stresses corresponding to the deflection $q_{i}(t)$ in each mode.

Numerical solutions for the response $q_{i}(t)$ of a linear oscillator to impacts $Q_{i}(t)$ of a variety of shapes are given in reference [1].

\section{Method of Separating Static Deflection and Vibrational Deflection (Williams)}

Williams outlined [2] a method of computing the displacement of an elastic structure as a result of applying an impact force of fixed distribution along the structure:

$$
F\left(x_{f}, t\right)=P(x) F(t),
$$

where

$$
\begin{aligned}
& P(x)= \text { spacial distribution of force, assumed } \\
& \quad \text { constant }
\end{aligned}
$$

Williams expands $P(x)$ in terms of the inertia loads of the various modes

$$
P(x)=\sum_{i=1,2, \ldots} L_{i}(x)
$$

where $L_{i}(x)$ will be proportional to the product of mass and amplitude at each station $m(x) \varphi_{i}(x)$. He reduces the number of natural modes $i$ that have to be included in the expansion by noting that the inertia forces become negligible compared to the elastic force for modes with sufficiently high frequency. The deflection in these higher modes is therefore the same as if the force were acting statically. The deflection in the lower frequency modes may be computed by adding a static force $L_{i}(x) f_{i}(t)$, equivalent to the inertia force, to the external force $L_{i}(x) F_{i}(t)$. Williams shows that the equivalent static force is given by

$$
L_{i}(x) f_{i}(t)=L_{i}(x) \int_{0}^{t} \cos \omega_{i}(t-\tau) \dot{F}(\tau) d \tau .
$$


The deflection of the structure under the impact load is then equal to that resulting from a static force,

$$
P(x) F(t)+\Sigma L_{i}(x) f_{i}(t) .
$$

It is interesting to note that a separation of the response into a static and a vibrational term can be made directly by integrating eq 3 by parts as follows:

$$
\begin{aligned}
q_{i}(t)= & \frac{1}{M \omega_{2}}\left[\left.Q_{i}(\tau) \frac{\cos \omega_{i}(t-\tau)}{\omega_{i}}\right|_{0} ^{t}-\right. \\
& \left.\int_{0}^{t} \dot{Q}_{i}(\tau) \frac{\cos \omega_{i}(t-\tau)}{\omega_{i}} d \tau\right] .
\end{aligned}
$$

Substituting limits and noting that

$$
\omega_{i}=\sqrt{K_{i} / M_{i}}
$$

gives

$$
q_{i}(t)=\frac{Q_{i}(t)}{K_{i}}-\frac{1}{M_{i} \omega_{i}{ }^{2}} \int_{0}^{t} \cos \omega_{i}(t-\tau) \dot{Q}_{i}(\tau) d \tau .
$$

The first term is the deflection of the equivalent linear oscillator of figure 1 on the assumption that the generalized force $Q_{i}(t)$ acts statically, and the second term is the vibrational deflection of the oscillator.

The resultant response of the structure is given by adding to the static deflection under the external load $F\left(x_{f}, t\right)$ the vibrational deflection in each mode. The solution is more general than that of Williams in not being restricted to external forces with a fixed distribution $P(x)$ in space. The two solutions coincide for the special case in which $F\left(x_{f}, t\right)$ can be written in the form of eq 6 .

\section{Method of Coupled Modes (Levy)}

Samuel Levy of this Bureau has suggested that it may be possible to represent the response to an impact in terms of fewer modes if the restriction to normal modes is dropped. He proposed a specific series of modes that can be computed directly for a structure with known influence coefficients.

Levy's method will be made clear by applying it to the case under discussion of computing the flexural transients in an airplane wing following the landing impact.

The rigid-body acceleration $\ddot{y}_{0}$ following the application of a unit impact force $P=1$ at a dis-

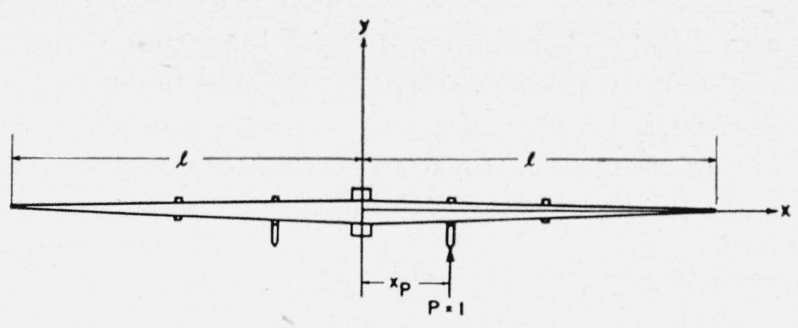

Figure 2. Force producing rigid-body translation and rotation of wing.

tance $x_{p}$ from the center of the wing in figure 2 is given by

where

$$
\ddot{y}_{0}=\frac{1}{M_{0}}+\frac{x_{p} x}{I}
$$

$$
\begin{aligned}
x= & \text { distance from center; } \\
M_{0}= & \text { mass of wing; } \\
I= & \text { polar moment of inertia of wing about } \\
& \text { longitudinal axis of airplane. }
\end{aligned}
$$

The shape $\phi_{1}(x)$ of the first vibrational mode is obtained by computing the deflection $y_{1}(x)$ caused by the combined action of the applied unit impact force and the distributed inertia force $\ddot{y}_{0} m(x)$, using the influence coefficients for rigid clamping of the wing at the center of gravity. The rigid-body motion is eliminated or "swept out" from $y_{1}(x)$, since it does not enter in the bending of the wing. The elimination is made by subtracting $a_{1}+b_{1} x$ from $y_{1}(x)$, where $a_{1}$ and $b_{1}$ are determined from the relations

$$
\begin{gathered}
\int_{-l}^{l}\left[y_{1}(x)-a_{1}\right] m(x) d x=0 ; \\
\int_{-l}^{l} x\left[y_{1}(x)-b_{1} x\right] m(x) d x+0 .
\end{gathered}
$$

$\phi_{1}(x)$ is taken as the deflection resulting after normalizing to give unit deflection at the tip on the side struck.

The second vibrational mode $\phi_{2}(x)$ is derived from the first by computing the deflection $y_{2}(x)$ resulting from inertia forces $m(x) \phi_{1}(x)$ and then subtracting $a_{2}+b_{2} x+c_{2} \phi_{1}(x)$, where $a_{2}, b_{2}$ and $c_{2}$ are given by

$$
\left.\begin{array}{l}
\int_{-l}^{l}\left[y_{2}(x)-a_{2}\right] m(x) d x=0, \\
\int_{-l}^{l} x\left[y_{2}(x)-b_{2} x\right] m(x) d x=0, \\
\int_{-l}^{l}\left[y_{2}(x)-a_{2}-b_{2} x-c_{2} \varphi_{1}(x)\right] L_{1}(x) d x=0,
\end{array}\right\}
$$


and $L_{1}(x)$ is the distribution of load that deflects the wing to the shape $\phi_{1}(x) . \phi_{2}(x)$ is taken as the normalized value of $y_{2}(x)-a_{2}-b_{2} x-c_{2} \phi_{1}(x)$. The last of these equations (14) expresses the condition that the load $L_{1}(x)$ should do no work on the deflections $\phi_{2}(x)$. The procedure for the derivation of modes of still higher order is obvious.

The "amplitudes" or generalized coordinates $q_{i}(t)$ in the vibrational modes are obtained by applying Lagrange's equations.

$$
\left.\begin{array}{l}
\frac{d}{d t}\left(\frac{\partial T}{\partial \dot{q}_{1}}\right)+\frac{\partial U}{\partial q_{1}}=\varphi_{1}\left(x_{P}\right) P(t) \\
\frac{d}{d t}\left(\frac{\partial T}{\partial \dot{q}_{2}}\right)+\frac{\partial U}{\partial q_{2}}=\varphi_{2}\left(x_{P}\right) P(t) \\
\ldots
\end{array}\right\}
$$

where $T, U$ are the kinetic energy and the potential energy, respectively, that are stored in the wing, and where $\phi_{i}\left(x_{P}\right) P$ is the generalized force acting on the wing in mode $i$. The energies $T, U$ may be computed by resolving the wing into a number of stations. The kinetic energy is then given by the sum of the kinetic energies of the masses $m_{n}$ at each station $n$ :

$$
T=\frac{1}{2} \Sigma_{n} m_{n}\left[\varphi_{1}\left(x_{n}\right) \dot{q}_{1}+\varphi_{2}\left(x_{n}\right) \dot{q}_{2}+\ldots\right]^{2} .
$$

The potential energy is given by the sum of the work done by the forces $q_{1} L_{1}\left(x_{n}\right), q_{2} L_{2}\left(x_{n}\right), \ldots$ at each station $n$ :

$U=\frac{1}{2} \Sigma\left[q_{1}^{2} L_{1}\left(x_{n}\right) \varphi_{1}\left(x_{n}\right)+q_{2}^{2} L_{2}\left(x_{n}\right) \varphi_{2}\left(x_{n}\right)+\ldots\right]$

Substituting these expressions for $T, U$ in eq 15 and carrying out the differentiation will lead to equations of the form

$$
\left.\begin{array}{l}
K_{1} q_{1}+M_{11} \ddot{q}_{1}+M_{12} \ddot{q}_{2}+\ldots=F_{1}(t) \\
K_{2} q_{2}+M_{12} \ddot{q}_{1}+M_{22} \ddot{q}_{2}+\ldots=0, \\
\ldots
\end{array}\right\}
$$

in which all quantities are known except the generalized coordinates $q_{1}, q_{2}, \ldots$. These equations may be solved by writing down separate equations for $\ddot{q}_{1}\left(q_{1}, q_{2}, \ldots\right), \ddot{q}_{2}\left(q_{1}, q_{2} \ldots\right)$, etc., and then solving these equations simultaneously by a numerical method such as Adams ${ }^{1}$ method [3, p. 363 to 367$]$.

\section{Method of Traveling Waves}

It was hoped to derive a method of traveling waves for computing flexural transients following a sharp impact on an airplane beam. Numerous attempts were made to develop such a method, but all these failed to lead to a procedure against which serious criticisms could not be raised. The chief difficulty in this problem as compared to St. Venant's problem of the longitudinal waves in an elastic bar of constant section seems to reside in the basic differential equation of the problem. In the case of St. Venant's problem, the equation of equilibrium of axial forces leads to $[4$, p. 200,285$]$

where

$$
\frac{\partial^{2} u}{\partial t^{2}}-\frac{E}{\rho} \frac{\partial^{2} u}{\partial x^{2}}=0
$$

$u(x, t)=$ displacement along axis of bar;

$E \quad=$ Young's modulus of material;

$\rho \quad=$ mass density of material.

The general solution of this equation can be written in the form

$$
u=f\left(\sqrt{\frac{E}{\rho}} t-x\right)+f_{1}\left(\sqrt{\frac{E}{\rho}} t+x\right),
$$

where, $f, f_{1}$ are arbitrary functions, which are determined by the initial conditions of the impact.

The solution given by eq 19 represents two traveling waves, one proceeding in the $+x$ direction and the other in the $-x$ direction. The velocity of propagation of the waves is $\sqrt{E / \rho}$, i. e., it depends on the properties of the material only.

A much more complicated differential equation applies in the case of flexural waves in an elastic bar of constant section. Equilibrium of transverse forces is expressed in this case, according to Timoshenko [4, p. 228], by the equation

$$
\begin{gathered}
E I \frac{\partial^{4} y}{\partial x^{4}}+\rho A \frac{\partial^{2} y}{\partial t^{2}}-\rho I \frac{\partial^{4} y}{\partial x^{2} \partial t^{2}}-\frac{E I \rho}{k^{\prime} G} \frac{\partial^{4} y}{\partial x^{2} \partial t^{2}}+ \\
\frac{\rho^{2} I}{k^{\prime} G} \frac{\partial^{4} y}{\partial t^{4}}=0
\end{gathered}
$$

where

$$
\begin{aligned}
y(x, t) & =\text { lateral deflection; } \\
E I & =\text { flexural rigidity of bar } ; \\
\rho A & =\text { mass per unit length of bar } \\
G & =\text { shear modulus } \\
k^{\prime} G= & \text { ratio of average shear stress to change } \\
& \quad \text { in slope produced by shearing force. }
\end{aligned}
$$


The first term in eq 20 is the distributed lateral force resisted by simple bending of the beam; the second is the force required to accelerate the sections of the beam in their lateral vibration; the third is that required to rotate the sections of the beam; and the last two are the forces required because of the shearing of the beam. The first two terms predominate over the last three in most beams:

$$
E I \frac{\partial^{4} y}{\partial x^{4}}+\rho A \frac{\partial^{2} y}{\partial t^{2}}=0 .
$$

This equation is of a different form than eq 18 . Equation 20 reduces to a simple wave equation of the type of eq 18 only for beams of abnormal proportions, in which the second term is negligible compared to other terms in the equation. It follows that a transverse impulse applied to an elastic beam of ordinary proportions will not travel along the beam without distortion, like a longitudinal impulse.

Nevertheless an attempt was made to solve the problem by a numerical method. The beam was replaced by a series of concentrated masses connected elastically by massless beam elements. It was attempted first to find the deflection of the masses following the application of a known impact force to one of the masses by writing eq 21 as a difference equation and then solving by numerical integration for the deflection at each station as a function of time. This attempt failed because too many stations along the beam were required to obtain sufficiently accurate values of $E I \partial^{4} y / \partial x^{4}$ at each station.

Next an attempt was made to solve the problem on the assumption that the impact was of sufficiently short duration that the response was confined to a small portion of the beam. A small number of equations can then be written to express the equilibrium between the inertia forces acting on the masses in the disturbed portion of the beam and the shearing forces transmitted by the beam elements to displace these masses relative to each other. This method of attack has been unsuccessful so far because of the difficulty of keeping the extent of the disturbed portion of the beam, outside of which all masses can be considered at rest, small enough so that only a few influence coefficients are required to give the relation between shearing forces and displacements.

\section{Solution for Soft Impact and Comparison with Experiment}

The first three methods outlined above were used to compute the bending strains introduced in a model wing following a soft vertical landing impact at two points. The impact was regarded as soft because the period of impact was about 1.7 times the fundamental flexural period of the wing. The model wing is shown in figure 3 . The wing was fabricated from aluminum alloy sheet and angles to have a distribution of flexural rigidity and of mass (figs. 4 and 5) similar to that of a large fourengine transport airplane. A "landing strut," $C$, was fastened below each one of the two inboard engine masses to transmit the landing impact to the wing. The landing struts contained a thinwalled tube to which wire strain gages were fastened to measure the landing impact force as a function of time.

The model was tested by releasing it in a nearly strain-free condition (see [5]) from a height of about 1 inch to make contact with two synthetic

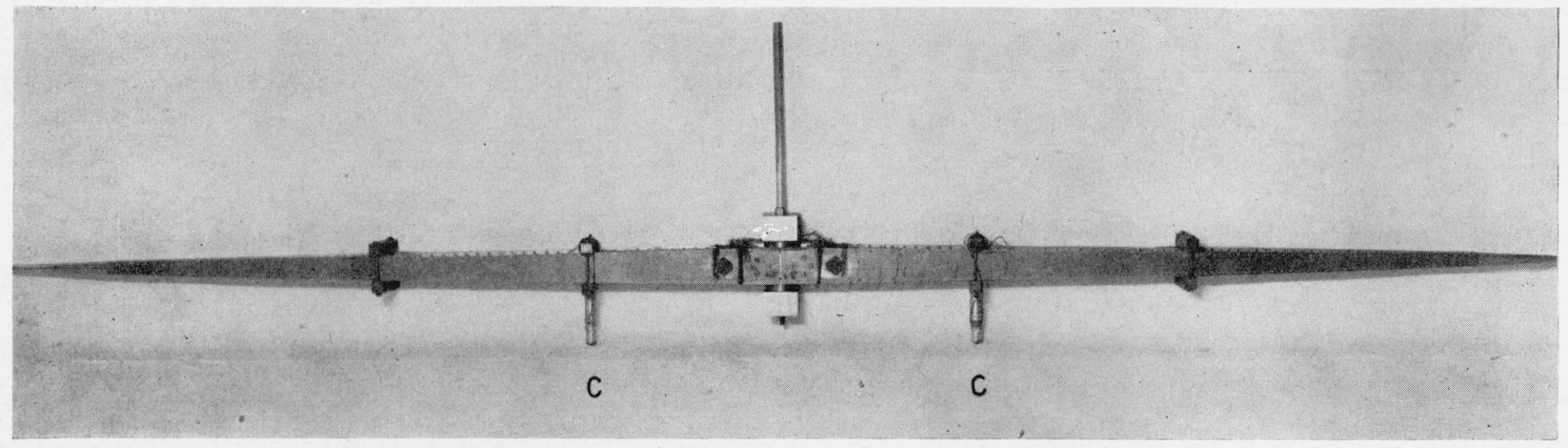

Figure 3. Model wing for two-point landing tests. 
"landing fields." The impact force was recorded by the dynamometers in the landing struts, and bending strains in the neighborhood of the inboard engines were recorded from wire strain gages by using the technique described in [5]. The shape of the landing-impact force was adjusted by using a suitable combination of rubber sheets and molding clay for the synthetic landing field. The time interval between contact at the two landing struts was adjusted by adjusting the relative heights of the two landing fields.

The computations were started by determining the first three symmetrical and the first three antisymmetrical flexural modes of the wing. For this purpose, the mass of the wing was considered to be concentrated at the root and at nine stations along each half of the wing. Influence coefficients between force and displacement at the mass points were computed by treating each half-wing as a

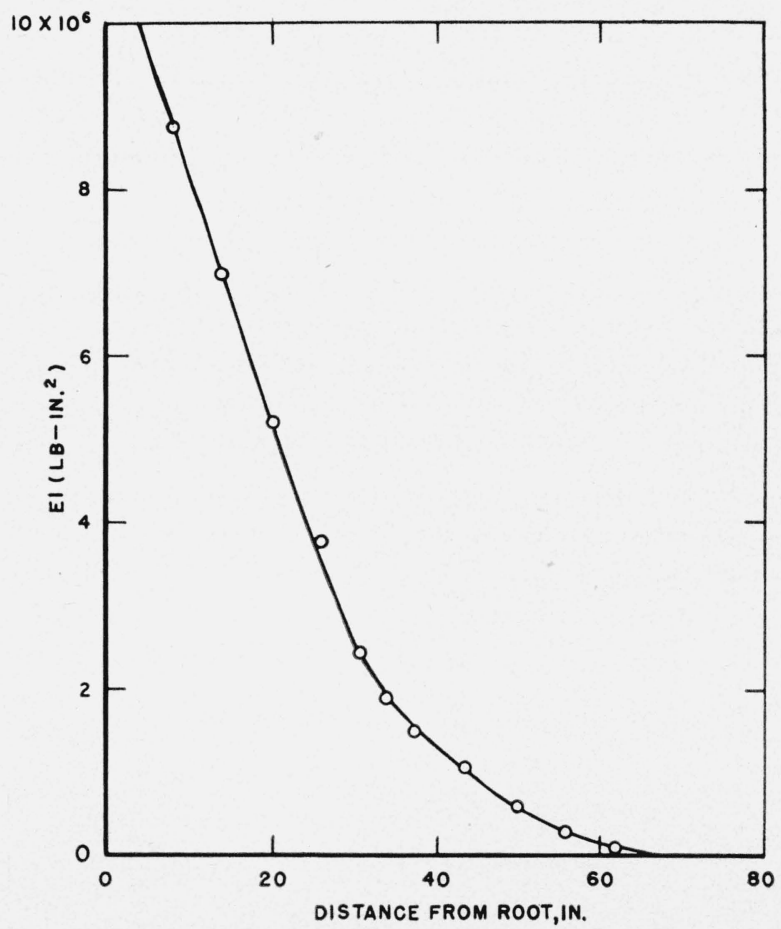

Figure 4. Flexural rigidity distribution for model wing

simple beam clamped at the root. The normal modes of the wing were obtained from the influence coefficients and from the given mass distribution by using a dynamic matrix and iteration procedure as explained by Duncan and Collar [6]. The deflection at the root of the half-wing (center of gravity of the wing) for the symmetrical modes was obtained from the condition that the center of gravity of the wing must remain at rest for freefree vibration. In the case of the antisymmetrical modes, the rotation at the root of the half-wing was obtained from the condition that the moments

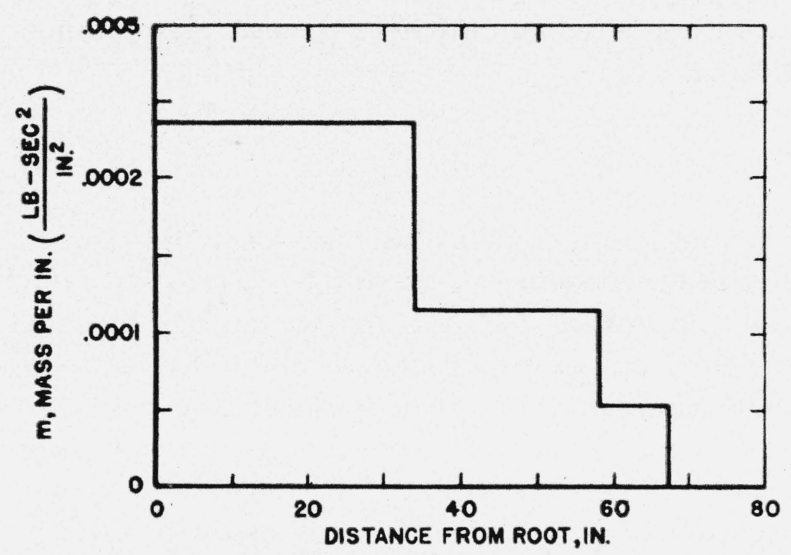

(a)

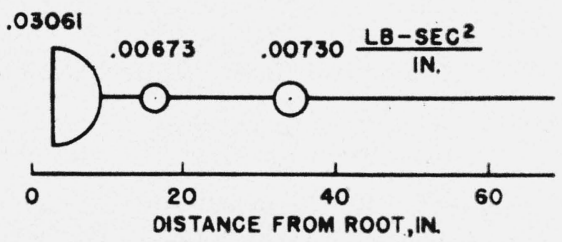

(b)

Figure 5. Mass distribution for model wing.

a, Distributed mass; $b$, concentrated mass.

about the center of gravity are zero for the freefree vibrations. The periods of the natural modes are given in table 1 . The first three coupled modes according to Levy's method were computed from the same influence coefficients as those used in the previous computations, following the procedure described in section II, 3 .

TABLE 1. Periods of computed natural flexural modes

\begin{tabular}{|c|c|}
\hline Mode & Period \\
\hline & Seconds \\
\hline First symmetrical & 0.0315 \\
\hline Second symmetrical_... & .0115 \\
\hline Third symmetrical & .00613 \\
\hline First antisymmetrical ...... & .0170 \\
\hline Second antisymmetrical & .00759 \\
\hline Third antisymmetrical ...... & $\because 00500$ \\
\hline
\end{tabular}


The response of the wing in each mode according to the normal-mode method and according to Williams' method was then computed by substituting the normalized modes and the generalized force obtained from the measured impact force at each landing strut in eq $2,3,8$, and 9 . The response according to Levy's method was obtained by solving eq 17 as indicated in section II, 3, substituting the given impact forces at the two landing struts and then superimposing the two solutions.

The bending moments in a wing section near the two landing struts were computed as a function of time from the amplitudes $q_{i}$ in each one of the component modes.

The resultant bending moments thus obtained are plotted against time in figure 6 . The figure also shows the bending moment corresponding to rigid body motion and the observed bending moment obtained from measurements of extreme fiber-bending strain in the two-point landing test.

It is apparent that the maximum values of the bending moments due to rigid-body motion amounted to about 85 percent of the maximum values of experimentally measured bending moments. The measured bending moments were in close agreement with those obtained by Levy's method and by Williams' method. It can be seen from figure 6 that the difference in maximum measured bending moments and maximum computed bending moments was less than 5 percent for Levy's method and less than 7 percent for Williams' method. For the method of normal modes, this difference between experimental and

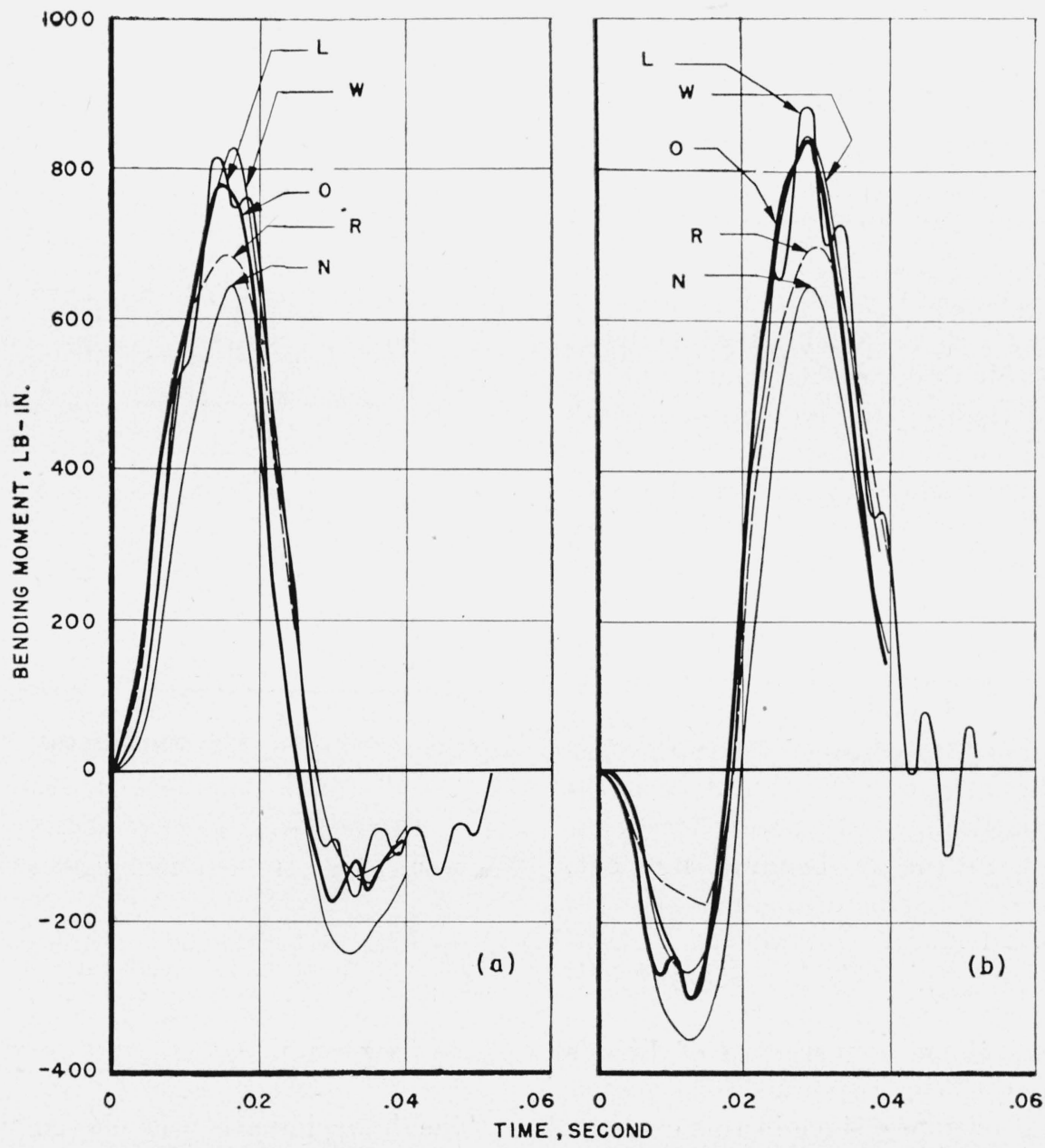

FIGURE 6. Response for soft, unsymmetrical, two-point landing impact.

a, Bending moment, 1 in. outboard of landing strut hitting first; b, bending moment, 1 in. outboard of landing strut hitting second. $O$, Observed; $N$, normal modes method; $W$, Williams' method; $L$, Levy's method; $R$, rigid body method. 


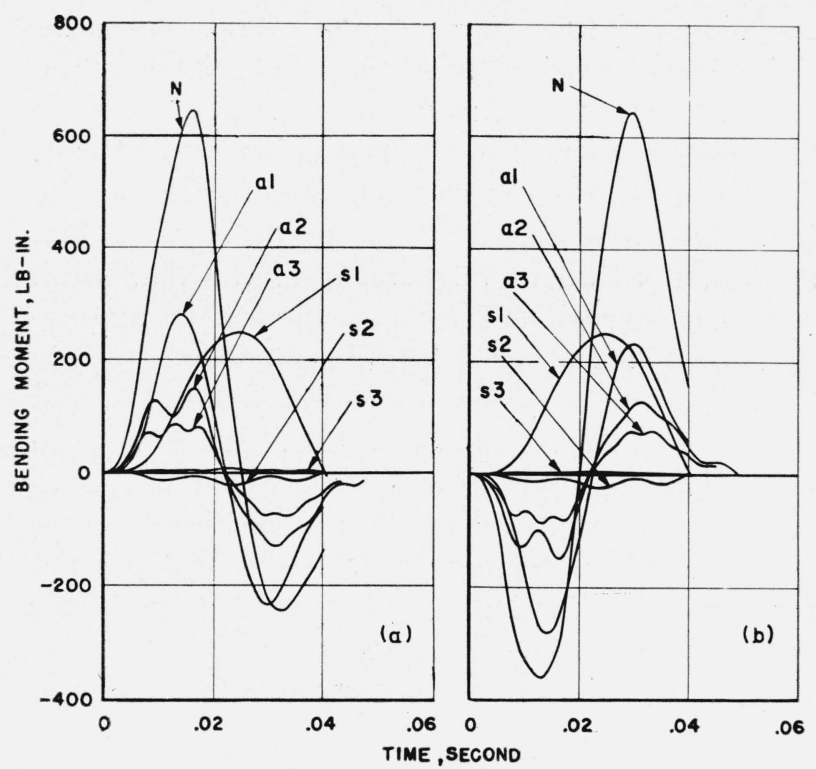

Figure 7. Components of response for soft, unsymmetical, two-point landing impact, normal modes method.

$\mathrm{a}$, Bending moment, $1 \mathrm{in}$. outboard of landing strut hitting first; $\mathrm{b}$, bending moment, 1 in. outboard of landing strut hitting second.

$\boldsymbol{N}$, Normal modes method (sum of the following components); s1, first symmetrical mode; s2, second symmetrical mode; s3, third symmetrical mode; a1, first antisymmetrical mode; a2, second antisymmetrical mode; a3, third antisymmetrical mode.

computed maxima was as much as 23 percent.

The reason for obtaining better agreement by Williams' method than by the method of normal modes becomes apparent from an examination of figures 7 and 8 , which show the contributions to the bending moment of the six different modes used in the computations. The normal modes method, figure 7 , shows good convergence for the symmetrical modes; however, the third antisymmetrical mode still contributes_ 13 percent to the measured maximum bending moment. Higher modes should probably be considered. In the case of Williams' method, figure 8, the third antisymmetrical mode contributes only about 1 percent to the measured maximum bending moment. The use of the first two symmetrical and the first two antisymmetrical modes in Williams' method would have sufficed to give the maximum bending moment within 8 percent.

It is difficult to judge convergence of Levy's method by comparing the response in the higher modes to the total response, since in this case the modes are coupled and the addition of a higherfrequency mode term changes the response in the lower-frequency modes. Hence the change in computed maximum bending moment due to the addition of a higher-frequency mode may be appreciably less than the response in that mode. Nevertheless, it is of interest to compare the response in the three modes. Figure 9 shows this response. The third mode contributed about 15 percent to the measured maximum bending moment.

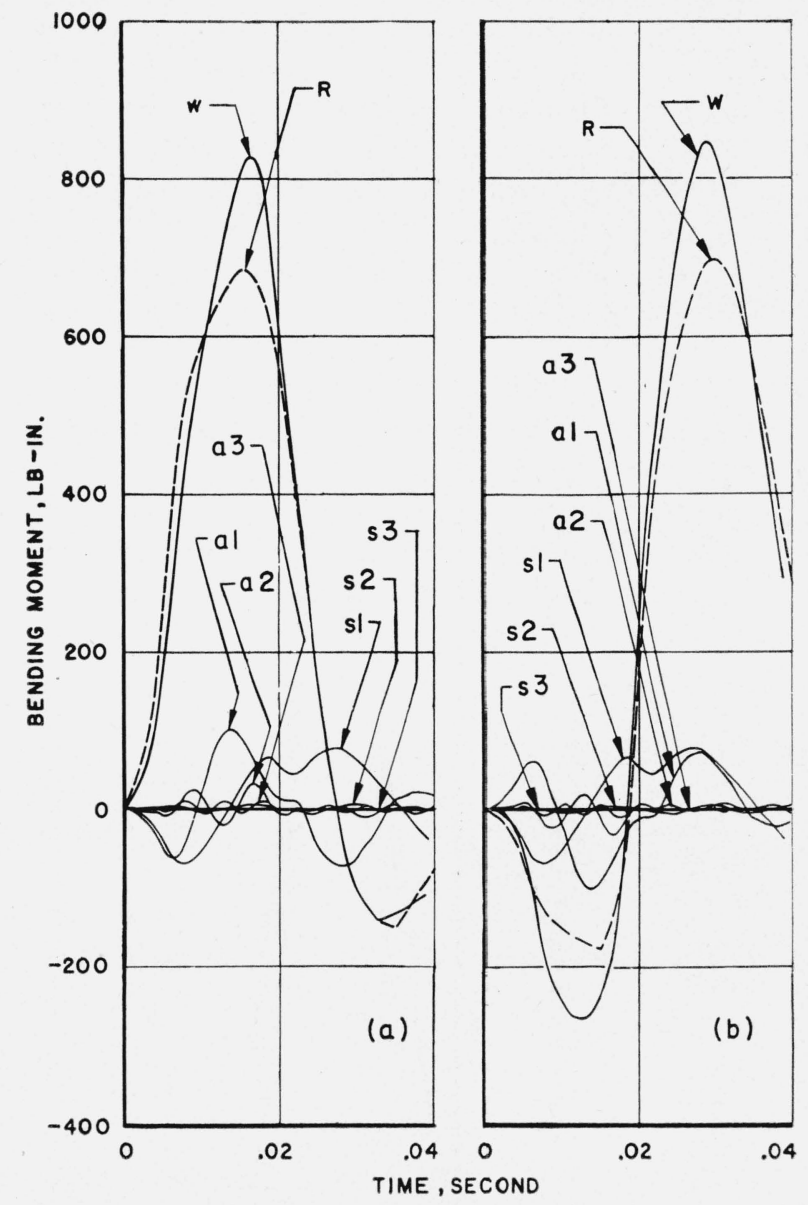

Figure 8. Components of response for soft, unsymmetrical, two-point landing impact, Williams' method.

a, Bending moment, 1 in. outboard of landing strut hitting first; $b$, bending moment, 1 in. outboard of landing strut hitting second.

$W$, Williams' method (sum of the following components); $R$, rigid body method; s1, first symmetrical mode; s2, second symmetrical mode; s3, third symmetrical mode; a1, first antisymmetrical mode; a2, second antis ym metrical mode; a3, third antisymmetrical mode.

\section{Solution for Hard Impact}

The hard impact was chosen as identical in shape to the soft impact, but the duration of the impact was reduced to one-fifth of that of the soft impact. The period of the impact was about 0.34 
times the fundamental flexural period of the wing. The response to the hard impact was computed keeping all quantities identical with those for the soft impact, except, of course, the duration of impact.

The resultant bending moment near the inboard engine above the landing strut, making first contact with the landing field, is given in figure 10 . The figure also shows the bending moment corressponding to the rigid body motion of the wing.

It is apparent that there is a larger divergence between the results of the three methods than for the soft impact and also that there is a considerable lag between the resultant response and the rigid body curve. The maximum bending moment according to the method of normal modes is 35 percent below that for the rigid body motion; that for Williams' method is 3 percent below, and that for Levy's method is 19 percent above.

The response in the individual modes of vibration according to the three methods is given in figures 11 to 13 . The convergence is good only for the symmetrical modes. The third antisymmetrical mode according to the method of normal modes contributes as much as 23 percent to the computed

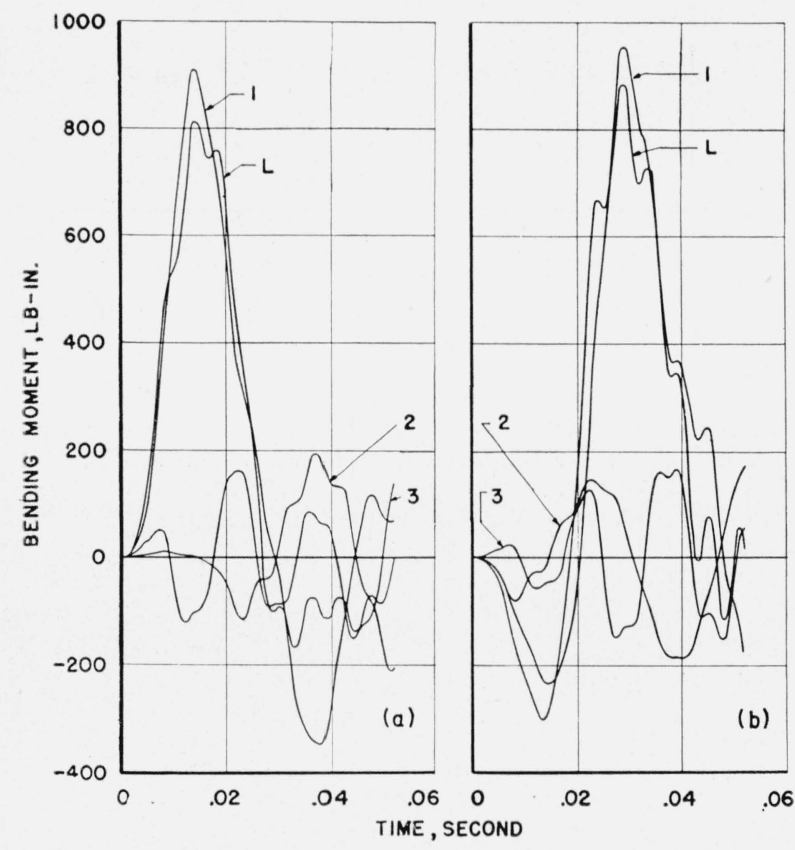

Figure 9. Components of response for soft, unsymmetrical, two-point landing impact, Levy's method.

a, Bending moment, 1 in. outboard of landing strut hitting first; $b$, bending moment, $1 \mathrm{in}$. outboard of landing strut hitting second.

$L$, Levy's method (sum of the following components); 1 , first mode; 2 , second mode; 3 , third mode.

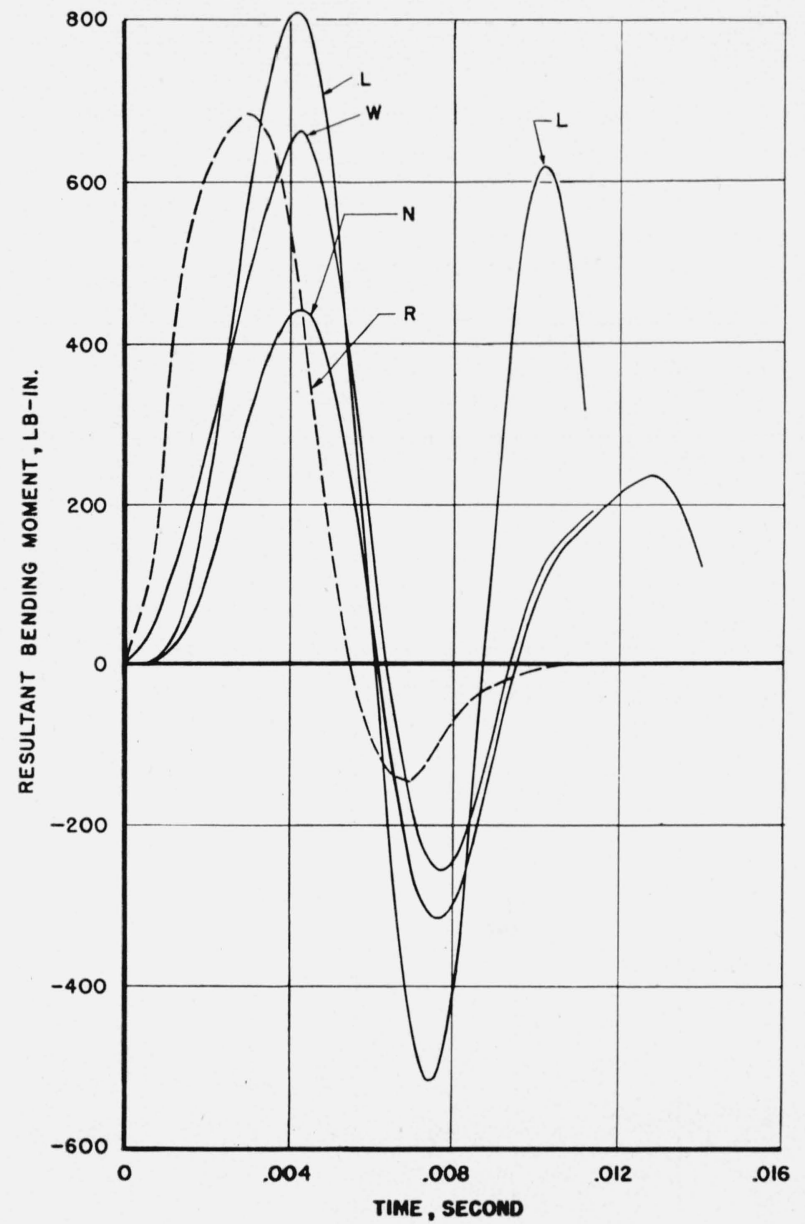

FIGURE 10. Response to hard, unsymmetrical, two-point impact.

Bending moment, 1 in. outboard of landing strut hitting first.

$N$, Normal modes method; $W$, Williams' method; $L$, Levy's method $R$, rigid body method.

maximum bending moment (fig. 11). For Williams' method, the third antisymmetrical mode contributes 15 percent to the computed maximum bending moment (fig. 12). For Levy's method, figure 13, the third coupled mode contributes 43 percent to the computed maximum total bending moment. It is apparent from this that a fourth analytical procedure needs to be developed to derive the response to impacts of very short duration, such as that chosen in the example.

\section{Conclusions}

Analysis of the flexural transients in a model airplane wing following a soft unsymmetrical twopoint landing impact indicates that Williams' method, separating static and vibrational response, 


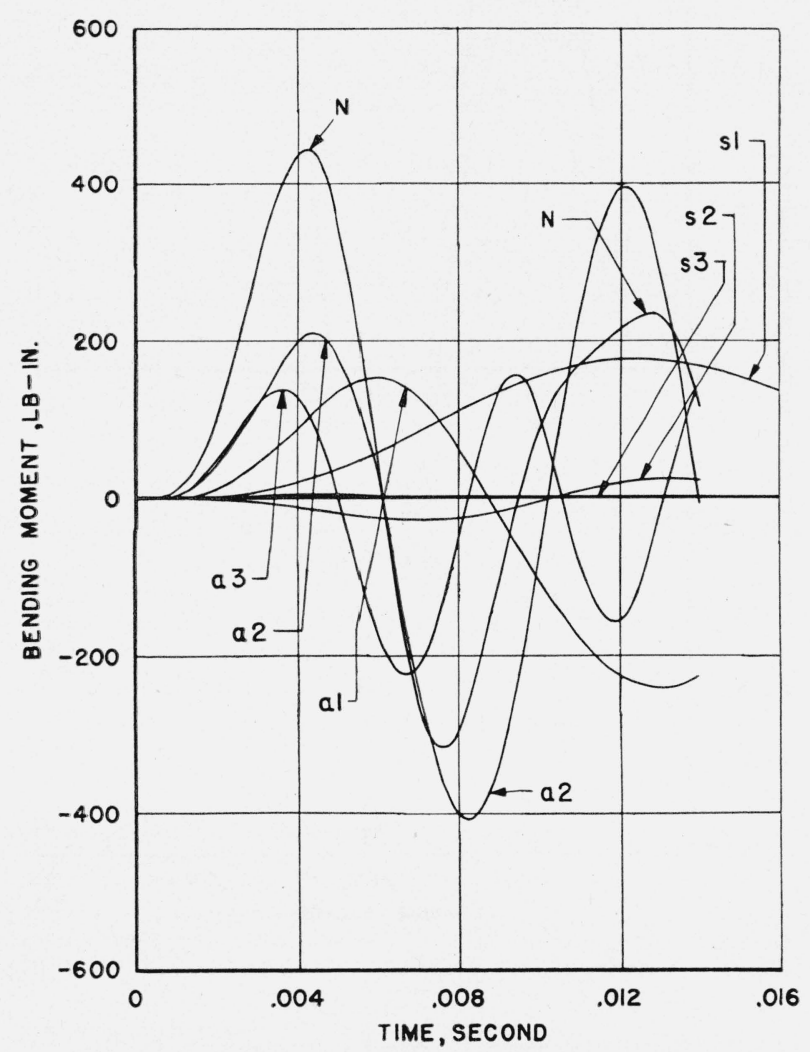

Figure 11. Components of response for hard, unsymmetrical, two-point impact, normal modes method.

Bending moment, 1 in. outboard of landing strut hitting first.

$N$, Normal modes method (sum of the following components); s1, first symmetrical mode; s2, second symmetrical mode; $\mathrm{s} 3$, third symmetrical mode; a1, first antisymmetrical mode; a2, second antisymmetrical mode; a3, third antisymmetrical mode.

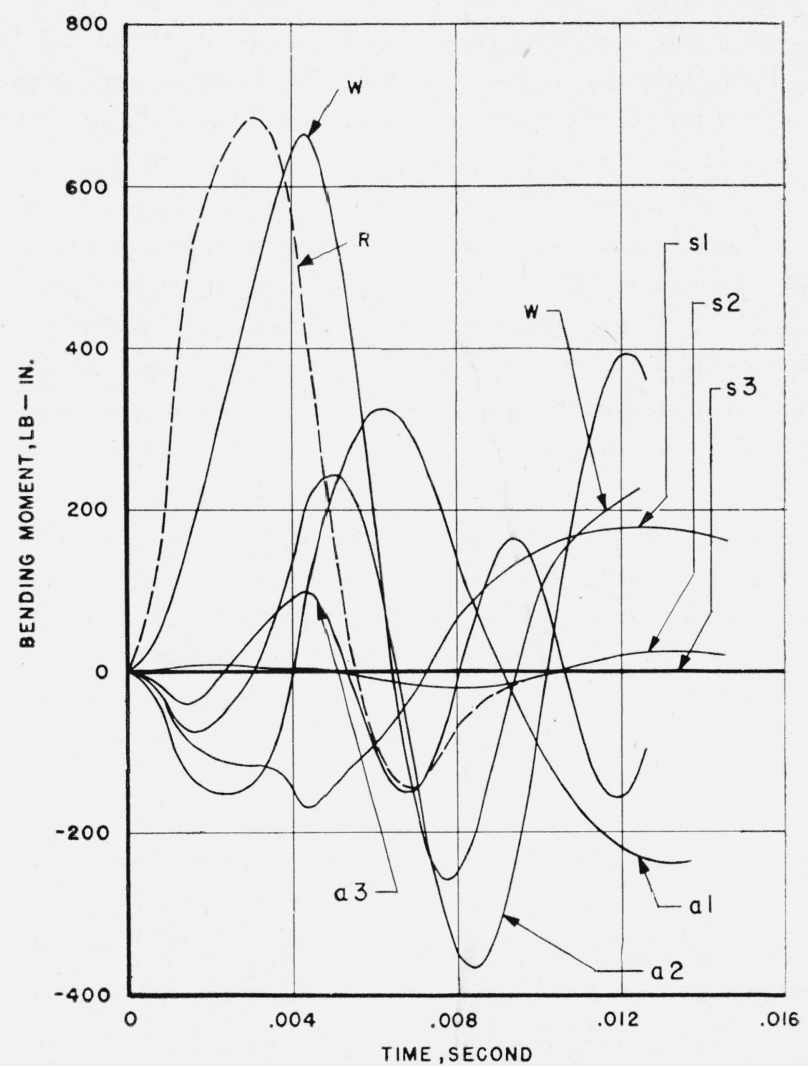

Figure 12. Components of response for hard, unsymmetrical, two-point impact, Williams' method.

Bending moment, 1 in, outboard of landing strut hitting first.

$W$, Williams' method (sum of the following components); $R$, rigid body method; s1, first symmetrical mode; s2, second symmetrical mode; s3, third symmetrical mode; a1, first antisymmetrical mode; a2, second antisyw. metrical mode; a3, third antisymmetrical mode. 


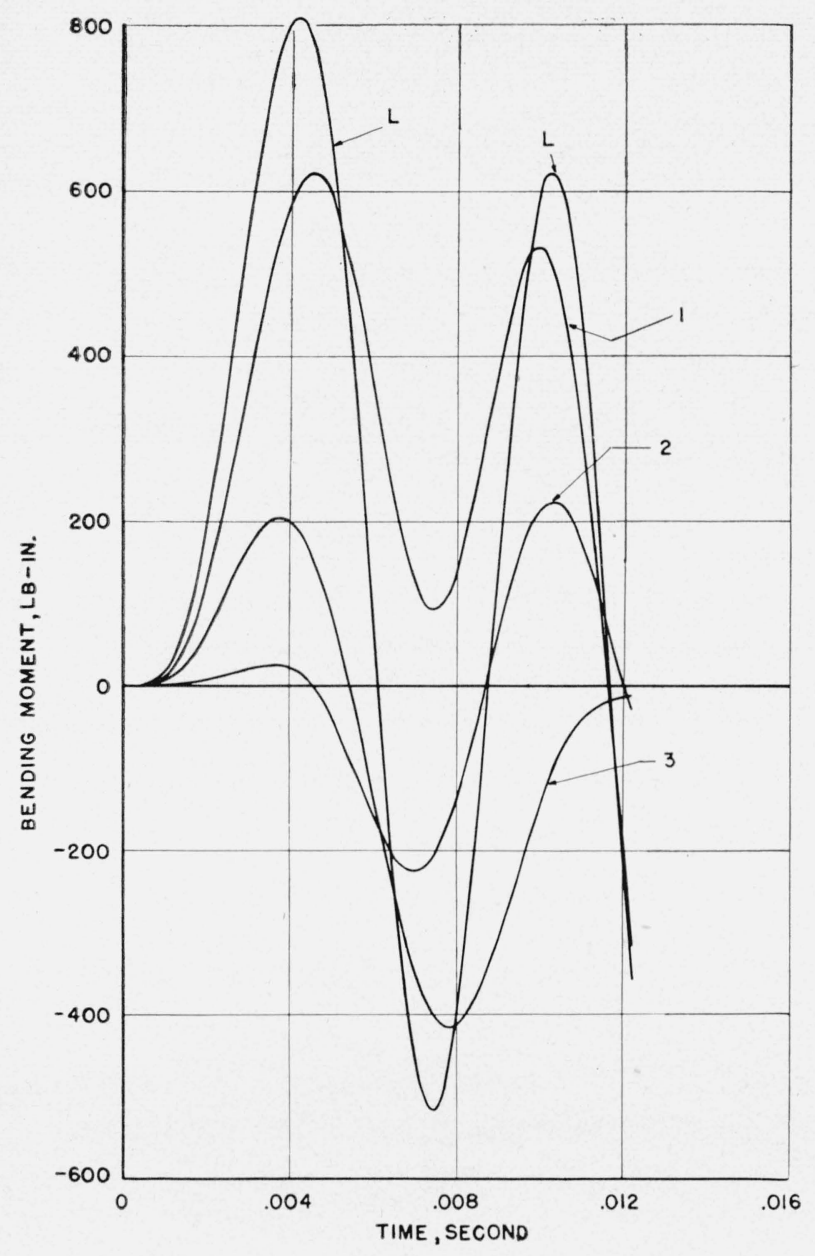

Figure 13. Components of response for hard, unsymmetrical, two-point impact, Levy's method.

Bending moment, 1 in. outboard of landing strut hitting first.

$L$, Levy's method (sum of the following components); 1 , first mode; 2, second mode; 3 , third mode.

is superior to the normal modes method used by Biot and Bisplinghoff, in leading to values in good agreement with experiment without using more than four modes. A method of coupled modes suggested by Levy leads to values of the response about as accurate as those from Williams' method with as few as three vibrational modes.

None of the three methods showed rapid convergence for the response following a hard impact of the same shape as the soft impact, but with only one-fifth of the duration. Attempts to develop a traveling-wave method for the solution of this problem have been unsuccessful so far.

The author expresses his indebtedness to his colleagues Samuel Levy, J. B. Woodson, and W. D. Kroll, who were principally responsible for the computation of the transients given in the paper.

\section{References}

[1] M. A. Biot and R. L. Bisplinghoff, Dynamic loads on airplane structures during landing, NACA Wartime Report W-92 (Oct. 1944).

[2] D. Williams, Displacements of a linear elastic system under a given transient load, Royal Aircraft Establishment SME C/7219/DW/19, August 1946. Presented before Sixth International Congress for Applied Mechanics, Paris, September 1946.

[3] E. T. Whittaker and G. Robinson, The calculus of observations, 4th ed. (Blackie \& Son, London, 1944).

[4] S. Timoshenko, Vibration problems in engineering (D. Van Nostrand Co., Inc., New York, N. Y., 1929).

[5] W. Ramberg and A. E. McPherson, Experimental verification of theory of landing impact, NBS J. Research 41, 509 (1948) RP 1936.

[6] W. J. Duncan and A. R. Collar, Solution of oscillation problems by matrices, Phil. Mag. [7] 17, 866 (1934).

Washington, June 3, 1948. 